\title{
Rhesus macaque MHC class I molecules show differential subcellular localizations
}

\author{
Cornelia Rosner • Philip H. Kruse • Torben Lübke • \\ Lutz Walter
}

Received: 9 December 2009 / Accepted: 18 January 2010/Published online: 12 February 2010

(C) The Author(s) 2010. This article is published with open access at Springerlink.com

\begin{abstract}
The MHC class I gene family of rhesus macaques is characterised by considerable gene duplications. While a $H L A-C$-orthologous gene is absent, the Мaтu- $A$ and in particular the Мати- $B$ genes have expanded, giving rise to plastic haplotypes with differential gene content. Although some of the rhesus macaque $M H C$ class I genes are known to be associated with susceptibility/resistance to infectious diseases, the functional significance of duplicated Мати- $A$ and Мати- $B$ genes and the expression pattern of their encoded proteins are largely unknown. Here, we present data of the subcellular localization of AcGFP-tagged Mamu-A and Mamu-B molecules. We found strong cell surface and low intracellular expression for Mamu- $A 1$, Maтu- $A 2$ and Мати$A 3$-encoded molecules as well as for Mamu-B*01704, Mamu-B*02101, Mamu-B*04801, Mamu-B*06002 and Mamu-B*13401. In contrast, weak cell surface and strong intracellular expression was seen for Mamu-A4*1403, Mamu-B*01202, Mamu-B*02804, Mamu-B*03002, MamuB*05704, Mamu-I*010201 and Mamu-I*0121. The different expression patterns were assigned to the antigen-binding $\alpha 1$ and $\alpha 2$ domains, suggesting failure of peptide binding is responsible for retaining 'intracellular' Mamu class I molecules in the endoplasmic reticulum. These findings indicate a diverse functional role of the duplicated rhesus macaque MHC class I genes.
\end{abstract}

\footnotetext{
C. Rosner $\cdot$ P. H. Kruse $\cdot$ L. Walter $(\bowtie)$

Abteilung Primatengenetik,

Deutsches Primatenzentrum - Leibniz

Institut für Primatenforschung,

Kellnerweg 4,

37077 Göttingen, Germany

e-mail: 1walter@gwdg.de

T. Lübke

Institut für Biochemie 2, Universität Göttingen,

Göttingen, Germany
}

Keywords MHC class I - Mamu-A · Mamu-B · Subcellular localization $\cdot$ Macaca mulatta

\section{Introduction}

Rhesus macaques are frequently used as important animal models for the study of human infectious and autoimmune diseases, in transplantation studies, and vaccine development. As is known from other species, also the rhesus macaque $M H C$ (Mamu) is associated with susceptibility and resistance to infectious and autoimmune diseases (Goulder and Watkins 2008; Sauermann et al. 2008; Vierboom et al. 2005). Significant progress has been made in the last years in the analysis of such disease associations, yet many of the various identified MHC class $I$ genes have not been functionally characterised. The reason for this may be that the rhesus macaque $M H C$ class I gene region is highly prone to gene expansions and contractions. While genes orthologous to $H L A-E$ and $H L A-F$ are present, rhesus macaques show extensive duplications of class $I$ genes that are assigned to the $M H C-A, M H C-B$ and $M H C-G$ lineages (Daza-Vamenta et al. 2004; Kulski et al. 2004; Otting et al. 2007; Otting et al. 2005). Four distinct Mamu- $A$ and $>20$ distinct Мати- $B$ genes were described in Indian rhesus macaques (Daza-Vamenta et al. 2004; Otting et al. 2007; Otting et al. 2005). A strict orthologue of $H L A-C$ is absent from the rhesus macaque $M H C$ and the duplicated $M a m u-G$ genes are pseudogenes (Daza-Vamenta et al. 2004). The function of $M a m u-G$ was likely replaced by duplicated Мати- $A G$ genes that show characteristics of both Maтu- $A$ and Mamu-G (Boyson et al. 1997). Further contributing to this complex genomic situation are highly variable numbers of class I genes in rhesus macaque MHC class I haplotypes, i.e., haplotypes show differential gene content. In addition, the classical class I gene Мати-Al exhibits considerable 
allelic polymorphism (Blasky et al. 2008; Karl et al. 2008), although too much smaller extent as compared to classical HLA class I genes. Blocks of repetitive elements in which the single class $I$ gene units are embedded (Bonhomme et al. 2007; Bonhomme et al. 2008; Kulski et al. 2004) substantially enhance unequal crossing-over events during meiosis, giving rise to differences in gene content. Such mechanisms are typical examples of 'birth and death' processes in MHC class I gene evolution (Nei et al. 1997).

Four and seven Мати- $A$ genes have been defined in rhesus macaques of Indian and Chinese origin, respectively (Blasky et al. 2008; Karl et al. 2008; Otting et al. 2007; Otting et al. 2005). These genes differ in their degrees of polymorphism and in transcriptional activity. Whereas alleles of the Mamu-Al gene were so far found on every haplotype, differential gene content is evident for Мати-A2, Мати-A3 and Maтu- $A 4$, which are less polymorphic and transcribed to lower extent (Otting et al. 2007). The Mamu-B subregion displays even a higher degree of complexity, as shown by genomic sequencing of a complete Mamu haplotype (DazaVamenta et al. 2004) and by cDNA cloning and segregation studies in rhesus macaque families (Otting et al. 2005; Otting et al. 2008). Based on promotor and exon/intron structure only 14 of the 19 Мати-B genes of the sequenced haplotype are expected to be transcribed, but only seven of them were found by cDNA analysis. These genes also include the socalled Maти-I genes that are members of the Мати- $B$ gene subfamily and were previously designated $М а т и-B^{*} 09$ (Urvater et al. 2000). Мати-I genes are characterised by low variability and low expression of their gene products on the cell surface upon transfection (Urvater et al. 2000). According to the number of clones found by cDNA cloning, the transcribed $М а т и-B$ genes are distinguished into 'major and minor expressors' (Otting et al. 2008).

Data on protein expression of certain Mamu-A and most Mamu-B molecules are largely lacking. Antigen presentation has been extensively studied for Mamu-Al alleles, in particular for allele Mamu-A1*001, which confers slow progression to AIDS and low viral loads upon experimental infection with the simian immunodeficiency virus (SIVmac). In addition, antigen presentation was described for Mamu$\mathrm{B}^{*} 001, \mathrm{~B}^{*} 003, \mathrm{~B}^{*} 008, \mathrm{~B}^{*} 012$ and $\mathrm{B}^{*} 017$ (Goulder and Watkins 2008; Hickman-Miller et al. 2005; Loffredo et al. 2007; Mothe et al. 2002; Voss and Letvin 1996). Here, we have established various expression constructs for Mamu-A and Mamu-B molecules with a carboxy-terminal AcGFP tag and studied their subcellular expression in MHC class I negative K562 cells by confocal laser microscopy and flow cytometry. We found classical surface expression for all Mamu-A molecules except Mamu-A4*01403 and divergent subcellular expression patterns for Mamu-B molecules. Our data indicate that some Mamu-B molecules are not loaded with peptides and therefore remain predominantly inside the cell.

\section{Materials and methods}

PBMC samples

Blood samples were obtained during regular veterinary inspections of 30 Indian rhesus macaques housed in the German Primate Centre. Peripheral blood mononuclear cells (PBMC) were obtained after ficoll gradient centrifugation for $45 \mathrm{~min}$

RNA isolation, cDNA synthesis, RT-PCR and cloning of MHC class $I$ and B2M cDNA

All rhesus macaque PBMC samples were pooled and total RNA was isolated with a Qiagen RNA Mini purification kit (Qiagen, Hilden, Germany). Complementary DNA (cDNA) was synthesised with oligo-dT primer and murine leukaemia virus reverse transcriptase (M-MLV-RT) (Promega, Mannheim, Germany). Polymerase chain reaction (PCR) was performed to amplify MHC class $I$ cDNA and rhesus macaque B2M (mmB2M) cDNA using BioTherm Taq DNA Polymerase (Genecraft, Cologne, Germany) with primer pairs for Mamu-A (Mamu-A-EcoRI-forward: GCTCGAATTCCAAGGATGGCGCCCCGAACCCT, Mamu-A-BamHI-reverse: GATAAGGATCCTCCACTTTA CAAGCCATAAGAGA), Mamu-B (Mamu-B-HindIIIforward: GCTCAAGCTTCGGTCATGCGGGTCA TGGCGCCCC, Mamu-B-KpnI-reverse: CGTAGGTACCT TAGCCGTGAGAGACACATCA), Мamu-I $\overline{\text { (Mamu-I- }}$ HindIII-forward: GCTCAAGCTTCGGTCATGCGGGT CATGGCGCCCG, Mamu-I-KpnI-reverse: CGTAGGTACC CAAGCTGTGAGAGACACATCAG), and $\overline{\mathrm{mmB} 2 \mathrm{M}}$ (mmB2M-forward: CGAGATGTCTCGCTCAGTG, mmB2M-reverse: TGACATGTCTCGATCCCACTT). HLA$C$-specific PCR was performed using primers HLA-C-HindIIIforward: CATAAGCTTCGAGATGCGGGTCATGGCGC and HLA-C-KpnI-reverse: GTAGGTACCTGGGCTTTA CAAGCGATGAGAGACT. Restriction sites introduced in primers for cloning of $M H C$ class I cDNA in the expression vector pAcGFP-N1 are underlined. PCRs were performed with a Labcycler (SensoQuest, Göttingen, Germany) and the following parameters were used: initial denaturation at $94^{\circ} \mathrm{C}$ for $4 \mathrm{~min}, 30$ cycles of $94^{\circ} \mathrm{C}$ for $60 \mathrm{~s}(30 \mathrm{~s}$ for $\mathrm{mmB} 2 \mathrm{M}), 60^{\circ} \mathrm{C}\left(62^{\circ} \mathrm{C}\right.$ for $\left.H L A-C\right)$ for $60 \mathrm{~s}(30 \mathrm{~s}$ for $\mathrm{mmB} 2 \mathrm{M})$ and $72^{\circ} \mathrm{C}$ for $90 \mathrm{~s}(60 \mathrm{~s}$ for mmB2M), followed by a final extension for $72^{\circ} \mathrm{C}$ for $5 \mathrm{~min}$. PCR products were analysed by agarose gel electrophoresis and purified with the Wizard ${ }^{\circledR}$ SV Gel and PCR Clean-Up System (Promega). MHC class I and mmB2M PCR products were cloned in the pGEM-T Easy vector (Promega) and pcDNA3.1/V5-His-TOPO TA expression vector (Invitrogen, Carlsbad, CA, USA), respectively. Isolated plasmids were sequenced with BigDye terminator cycle sequencing 
chemistry and samples were analysed in an ABI 3130xl automatic sequencer (Applied Biosystems, Foster City, CA, USA). MHC class I cDNA clones with identical sequences that were found at least three times were used for subcloning in the pAcGFP-N1 expression vector (Clontech, Carlsbad, CA, USA).

Establishment of chimeric and mutated Mamu class I constructs

Chimeric constructs of Мати-I*010201 and Мати$B^{*} 06002$ were established by $\mathrm{PCR}$, exchanging either exon 2 or exon 3 or both exons. Forward and reverse primers at the ends of exons 2 and 3 were constructed $(\alpha 1$ exchange F: GGGTCTCACACCCTCCAG; $\alpha 1$ exchange R: GGAGGGTGTGAGACCCC; $\alpha 1+\alpha 2$ exchange F: GGATCCCCCAAAGACACAC; $\alpha 1+\alpha 2$ exchange R: CTTTGGGGGATCCGCGC). 5' and $3^{\prime}$ part were amplified from Мати-I*010201-AcGFP and Мати-B*06002AcGFP constructs with one internal primer and one vector primer (pAcGFP F: CAAATGGGCGGTAGGCGTG or pAcGFP R: GTGGCCATTCACATCGCCAT) with proofreading Pfu DNA polymerase (Fermentas, Ontario, Canada). For the exchange of the $\alpha 2$ domain, the $\alpha 1$-exchanged chimeric constructs were used as templates together with the primers for the $\alpha 1+\alpha 2$ exchange. PCR products were isolated after gel electrophoresis and used as templates for the generation of chimeric constructs in a further PCR: five cycles without and 25 cycles with pAcGFP vector primers. Obtained PCR products were digested with restriction enzymes EcoRI and KpnI and ligated into EcoRI/KpnIdigested pAcGFP-N1 expression vector.

To establish untagged rhesus macaque MHC class I molecules, the AcGFP tag was removed by site-directed mutagenesis of the AcGFP start codon (AUG) into a stop codon (UGA) in Mamu-A1*00101, Maтu-A1*00801, Мати-A1*01101, Мати-A2*050402, Мати-A3*1311, Мати-A4*1403, Мати-B*01202, Мати-B*02101, Мати$B^{*} 02804$, Мати- $B^{*} 06002$, Мати-I*010201 and Мати$I^{*} 0121$ expression constructs. Primers introducing the amino acid change (without AcGFP F: CGGGATCCACCGGTCT GAGTGAGCAAGGGCGCC; without AcGFP R: GGCGCCCTTGCTCACTCAGACCGGTGGATCCCG) were used with the QuikChange Multi Site-Directed Mutagenesis Kit according to the manufacturer's recommendations (Stratagene, La Jolla, CA, USA). All chimeric or mutated constructs were finally controlled by sequencing analysis.

Transfection of K562 cells, confocal microscopy and flow cytometry

K562 cells (human erythromyeloid leukaemia, DSMZ, Germany) were propagated in DMEM medium (PAA,
Pasching, Austria), supplemented with 10\% inactivated FCS, $100 \mathrm{U} / \mathrm{ml}$ penicillin and $0.1 \mathrm{mg} / \mathrm{ml}$ streptomycin (PAA). Cells were split 1:3 to 1:5 every second or third day. Cells were routinely tested for presence of mycoplasma using $1 \mu \mathrm{l}$ of cell culture supernatant as template in a PCR with primers MYCF1: ACACCATGGGAGCTGGTAAT and MYCR1: CTTCWTCGACTTYCAGACCCAAGG CAT followed by a nested PCR with primers MYCF2: GTTCTTTGAAAACTGAAT and MYCR2: GCATCCAC CAWAWACTCT.

K562 cells were also transiently transfected using Metafectene reagent according to manufacturer's instructions (Biontex, Martinsried, Germany). Transfection efficiency was controlled $48 \mathrm{~h}$ later by fluorescence microscopy via AcGFP fluorescence.

K562 cells were transiently co-transfected with Mamu-B and rhesus macaque $B 2 M$ constructs as described above. Expression of V5-tagged B2M was controlled in parallel experiments with mouse anti-V5 monoclonal antibody (Invitrogen) and goat anti-mouse IgG-PE-Cy5 polyclonal antibody (Santa Cruz Biotechnology, Santa Cruz, CA, USA).

Surface expression of MHC class I molecules was verified by confocal microscopy and flow cytometry. Cells $\left(5 \times 10^{5}\right)$ were washed twice with phosphate-buffered saline $(1 \times$ PBS $)$ and stained with the anti-MHC class I monoclonal antibody W6/32 (hybridoma HB-95, ATCC) or the anti-B2M monoclonal antibody BBM.1 (sc-13565, Santa Cruz Biotechnology) for $1 \mathrm{~h}$ at $4^{\circ} \mathrm{C}$. Binding of W6/32 or BBM.1 was detected with a goat anti-mouse IgGPE-Cy5 polyclonal antibody (SC-3799, Santa Cruz Biotechnology). For microscopy, cells were resuspended in $30 \mu \mathrm{l} 1 \times$ PBS and $10 \mu \mathrm{l}$ were put on a slide and covered with a cover slip. Pictures were taken immediately to ensure that cells were still alive. All images were collected with confocal laser scanning microscope LSM 5 PASCAL (Zeiss) through $\times 63$ objectives. Images were processed with Photoshop CS software (Adobe, San Jose, CA, USA).

For flow cytometry, cells were resuspended in $200 \mu \mathrm{l}$ of $1 \times$ PBS and 30,000 events were measured in a LSR II flow cytometer (BD Bioscience, San Jose, USA). Living cells were gated based on forward and side scatter characteristics. Data were acquired with BD FACS Diva 5.1 Software (Becton Dickinson) and subsequently analysed with FlowJo 5.7.1 or 7.5.5 Software (TreeStar, Ashland, OR, USA).

Co-localisation of AcGFP-tagged Mamu-A and Mamu-B molecules with organelle-specific marker molecules

K562 cells were transiently transfected with Мamu$A 1^{*} 00101$ or Mamu- $B^{*} 01202$ expression constructs and grown for $48 \mathrm{~h}$ in $5 \% \mathrm{CO}_{2}$ and $37^{\circ} \mathrm{C}$. Cells were harvested 
and washed twice with $1 \times$ PBS followed by fixation with $2 \%$ paraformaldehyde for $10 \mathrm{~min}$ at room temperature. After two rounds of washing with $1 \times$ PBS, cells were permeabilised with $0.5 \%$ Triton $\mathrm{X}-100$ in $1 \times$ PBS for $5 \mathrm{~min}$ at room temperature. Non-specific binding was blocked by incubation with $5 \%$ bovine serum albumin (BSA) in $1 \times$ PBS for $30 \mathrm{~min}$ at room temperature. Antibodies were diluted in $0.5 \%$ BSA in $1 \times$ PBS for detection of organelle markers; primary antibodies were applied overnight at $4{ }^{\circ} \mathrm{C}$ (anti-Calnexin, 1:1,000 (BD Bioscience); anti-GM130 1:250 (BD Bioscience); and anti-LAMP-1 1:25 (clone: H4A3, Developmental Studies Hybridoma Bank, Iowa City, IA, USA)). After washing twice with $1 \times$ PBS, antibody staining was visualised by incubation with a secondary antibody (polyclonal rabbit anti-mouse-TRITC, DAKO, Glostrup, Denmark; 1:40) for $1 \mathrm{~h}$ at room temperature. Cells were washed twice with $1 \times$ PBS followed by mounting with fluorescent mounting medium (DAKO). Fluorescent cells were analysed with a confocal laser scanning microscope LSM 5 PASCAL (Zeiss) through $\times 63$ objectives.

\section{Nomenclature and database accession numbers}

Novel cDNA sequences were submitted to DDBJ/EMBL/ GenBank database: Mamu-B*01202 (GQ387655), Mamu-

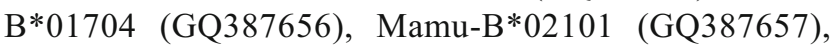
Mamu-B*05704 (GQ387658), Mamu-B*02801 (GQ387659), Mamu-B*13401 (GQ387661), MamuI*0121 (GQ387662). Sequences were also submitted to the Immunopolymorphism Database IPD (Robinson and Marsh 2007) and received official designations (http:// www.ebi.ac.uk/ipd/mhc/). As in the meantime numbers of known Maтu- $A 1$ alleles and Мати- $B$ sequences both exceed 100 , the Mamu-A1 allelic lineages and the various Maтu- $B$ sequences are distinguished by the first three digits after the star, e.g., Мати-A1*001 or Мати- $B^{*} 012$, which were previously designated $M a т u-A 1 * 01$ (or MamuA01), and $М а т и-B^{*} 12$.

\section{Results and discussion}

We isolated Mamu-A and Mamu-B cDNAs from a pool of 30 rhesus macaques of Indian origin by RT-PCR cloning. Altogether, 17 different cDNAs were identified, of which six belong to the Mamu- $A$ and 11 to the $M a m u-B / M a m u-I$ lineages. Sixteen of these cDNA sequences were identical (or nearly identical) to already published Мати- $A$ or Maтu-B/Mamu-I sequences. One cDNA sequence was less than $96 \%$ identical to any of the known rhesus macaque $M H C$ class $I$ sequences, likely representing a new gene or allele that received the designation Mamu- $B^{*} 13401$ from the Immuno Polymorphism Database (IPD-MHC database). We were interested in studying the cell surface expression of the various Mamu- $A$ and $M a m u-B$-encoded molecules. As reactivity of many rhesus macaque MHC class I proteins with mouse anti-human MHC class I monoclonal antibody W6/32 is not known, we fused the MHC class I cDNA sequences with $A c G F P$ for visualisation in K562 cells. As controls we included K562 cells transfected with AcGFP and AcGFP-tagged HLA-Cw*0303, respectively.

\section{Expression patterns of Mamu-A and Mamu-B molecules}

Six Mamu-A molecules were included in our analysis: three alleles of the polymorphic classical MHC class I gene Mamu-A1 $\left(A 1^{*} 00101, A 1^{*} 00801, A 1^{*} 01101\right)$ and one allele each of the less polymorphic Mamu-A2 (A2*050402), Mamu-A3 $\left(A 3^{*} 1311\right)$ and Mamu-A4 $\left(A 4^{*} 1403\right)$ genes. Strong cell surface expression was observed with monoclonal antibody W6/32 for the three Maтu-A1 alleles and for $M a m u-A 3^{*} 1311$ with a mean fluorescence intensity (MFI) between 540 and 843 (Fig. 1a) and to lower extend for Maтu-A2*050402 (MFI: 326). Мати-A4*1403 revealed the lowest surface expression (MFI: 105) among all Mamu-A molecules. The same tendency in MFI values was observed upon staining of the cells with the anti- $\beta 2$ microglobulin antibody BBM.1 (Fig. 1b), indicating that lower MFI values reflect fewer MHC class I molecules on the cell surface and not lower affinity of W6/32 antibody for different Mamu-A molecules. Confocal microscopy of the AcGFP-tagged Mamu-A molecules demonstrated that $A 1, A 2$ and $A 3$-encoded molecules are mainly found on the cell surface, and only few molecules were detected inside the cell (Fig. 1a). In contrast, Mamu-A4*1403 is found mainly inside the cell. Notably, cell surface expression pattern of the studied Mamu-A molecules roughly correlates with known transcription levels of Mamu-Al (high), Mamu-A2 and Maтu-A3 (medium) and Mamu-A4 (low)

Fig. 1 Analysis of Mamu-A and Mamu-B protein expression. a K562 cells were transiently transfected with expression constructs encoding AcGFP-tagged Mamu-A, Mamu-B or HLA-Cw*0303 or empty AcGFP vector. The latter two served as controls. Confocal microscopy: left panels show AcGFP fluorescence, middle panels show binding of monoclonal mouse anti-MHC class I antibody W6/32, right panels show merge. Flow cytometry: green lines show binding of W6/32, dotted grey lines are negative controls (empty AcGFP vector) and dashed grey lines show secondary antibody control (polyclonal PeCy5-coupled goat anti-mouse antibody). The mean fluorescence intensity (MFI) is given in the upper right corner. Results show one of at least three independent experiments. b AcGFPtagged Mamu-A and Mamu-B expression constructs were transiently transfected in $\mathrm{K} 562$ and tested for binding of anti-MHC class I monoclonal antibody W6/32 and anti-beta-2-microglobulin antibody BBM.1 in flow cytometry. Relative binding is indicated as ratio of MFI (W6/32 or BBM.1)/MFI (mock transfection). The average of three independent experiments is shown and error bars denote SEM 


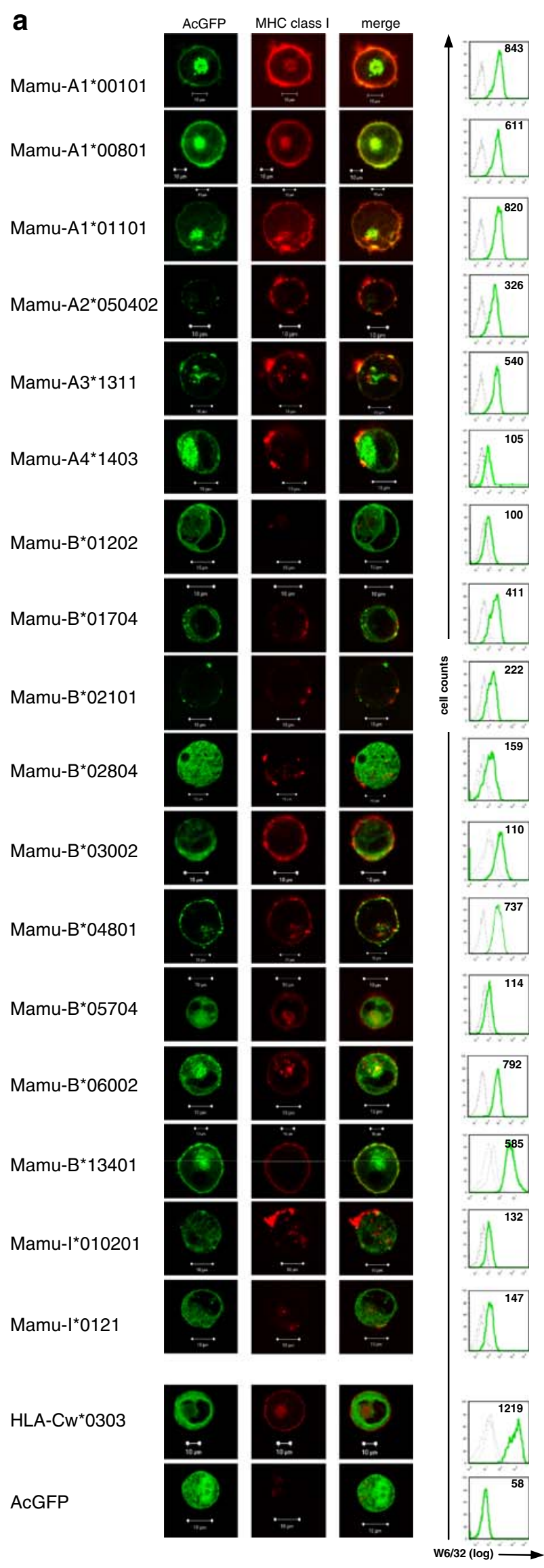

b relative binding of $W 6 / 32$ and BBM.1

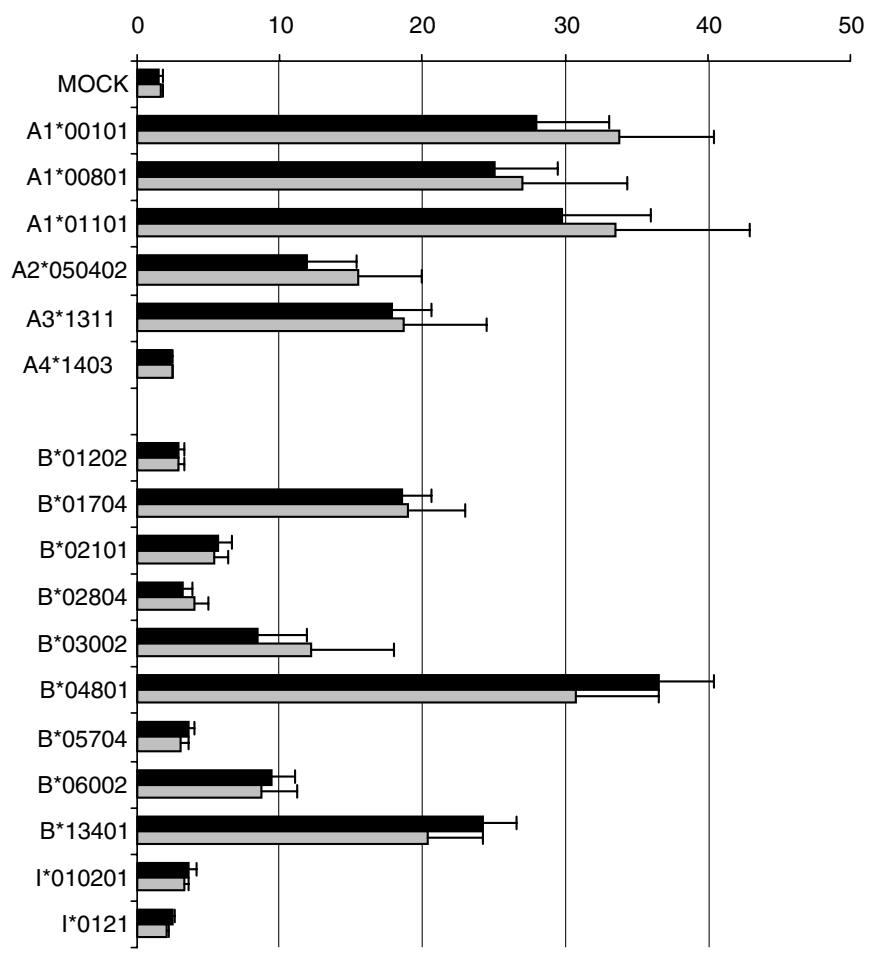


according to data published by Otting and colleagues (2007).

From our cDNA pool we cloned nine Мати- $B$ and two Mamu-I cDNAs. Only one of these cloned Мати- $B$ cDNA sequences $\left(\right.$ Mamu- $\left.B^{*} 03002\right)$ is identical with a gene of the completely sequenced MHC class I haplotype (accession no. AB128049; Мати-B5). All other cloned sequences are either alleles from already known Мamu- $B$ genes or represent distinct genes. Analysis of the various Мати- $B$ transfected K562 cells by flow cytometry and confocal microscopy also revealed different protein expression patterns. Among all Mamu-B transfectants, $\mathrm{B}^{*} 01704, \mathrm{~B}^{*} 02101$, $\mathrm{B}^{*} 04801, \mathrm{~B}^{*} 06002$ and $\mathrm{B}^{*} 13401$ showed the highest level of cell surface expression according to binding of W6/32, with MFI values ranging between 222 and 792 (Fig. 1a). Cell surface expression of these Mamu-B molecules is comparable to Mamu- $A$-encoded class I molecules (except Mamu-A4*1403) regarding quantity (MFI range) and quality (confocal microscopy). Also similar to Mamu-A molecules is a rather low level of intracellular expression of these MamuB molecules, although $\mathrm{B} * 01704$ and $\mathrm{B} * 06002$ display some intracellular AcGFP fluorescence. A second group of MamuB molecules, B*01202, B*02804, B*03002, B*05704, $I^{*} 010201$ and $I^{*} 0121$ are characterised by weak surface expression with MFI values ranging between 100 and 159 and strong intracellular expression similar to what was found for Mamu-A4*1403 (Fig. 1a). The same tendency in all Mamu-B MFI values was observed upon staining of the cells with the anti- $\beta 2$-microglobulin antibody BBM.1 (Fig. 1b). Thus, $B^{*} 01704, B^{*} 02101, B^{*} 04801, B^{*} 06002$ and $B^{*} 13401$ show protein expression levels similar to classical class $I$ molecules and $B^{*} 01202, B^{*} 02804, B^{*} 03002, B^{*} 05704$, $I^{*} 010201$ and $I^{*} 0121$ to non-classical ones.

Otting and colleagues (2005) distinguished Мати-B genes as 'majors' and 'minors' according to mRNA transcription levels and speculated that they are classified accordingly as classical and non-classical MHC class I genes. The classification as 'major' (classical class I) only partially overlaps with our protein expression data: $B^{*} 01704, B^{*} 02101, B^{*} 04801$ and $B^{*} 06002$. However, we also found disagreeing results as $B^{*} 01202, \mathrm{~B}^{*} 02804$ and $B^{*} 03002$ are classified as 'majors' with respect to transcription, but are characterised by a very low cell surface and mainly intracellular expression pattern more compatible with a non-classical MHC class I protein. This incongruence might be due to post-transcriptional and post-translational regulatory mechanisms such as RNA interference, inefficient protein translation, or increased protein degradation, respectively. Hence, mRNA transcription level might not necessarily be a highly reliable predictor of cell surface protein expression or classical/non-classical characters.

Although we did not analyse multiple alleles of single rhesus macaque $M H C$ class I genes, we do not expect allelic differences to drastically change the protein expression pattern. This assumption is based on the fact that $M H C$ class $I$ allelic polymorphism is generally low in rhesus macaques (Otting et al. 2005), but also reflects to a certain extent our limited knowledge of many Мати-B sequences whether they represent alleles or distinct genes. Therefore, we cannot exclude the possibility that allelic differences are indeed associated with changes of cellular expression patterns.

Intracellular localization of rhesus macaque class I molecules is due to ER retention

The construction of GFP fusion proteins is a wellestablished method to visualise expression and to determine subcellular localization of target molecules, especially if specific antibodies are not available. In previous studies, GFP-tagged HLA class I proteins were shown to behave in a similar manner as their wild-type counterparts (Gromme et al. 1999). Nevertheless, to exclude any potential effect of the AcGFP tag, a stop codon was introduced by sitedirected mutagenesis in expression constructs $A 1^{*} 00101$, $A 1 * 00801, A 1 * 01101, A 2 * 050402, A 3 * 1311, A 4 * 1403$, $B^{*} 01202, B^{*} 02101, B^{*} 02804, B^{*} 06002, I^{*} 010201$ and $I^{*} 0121$. No significant differences between tagged and untagged Mamu class I molecules were observed (Fig. 2a), ruling out a negative effect of AcGFP-tagging on MHC class I cell surface expression. Availability of $\beta 2-$ microglobulin is a regulating factor of cell surface expression of many, but not all MHC class I molecules (Myers et al. 1996). To rule out that inefficient binding of human $\beta 2$-microglobulin and unavailability of rhesus macaque $\beta 2$-microglobulin leads to lower expression levels of some Mamu-B molecules on the surface of human K562 cells, we transiently co-transfected rhesus macaque $B 2 M$ along with two high expressing $\left(B^{*} 01704, B^{*} 06002\right)$ and three low expressing constructs $\left(B^{*} 01202, B^{*} 02804\right.$, $\left.I^{*} 010201\right)$. As is shown in Fig. 2b, presence of rhesus macaque $\beta 2$-microglobulin has no significant effect on cell surface expression of all studied Mamu-B molecules. Thus, we can exclude the AcGFP tag and unavailability of rhesus macaque B2M as influencing factors of variable Mamu-B expression patterns in K562 cells.

Peptide receptive class I molecules are usually retained in the ER (Bijlmakers and Ploegh 1993) due to improper folding, lack of glycosylation, or failure of peptide loading. However, misfolded or empty class I molecules are not completely retained in the ER, they can cycle between the cis-Golgi, the intermediate compartment and the ER (Garstka et al. 2007; Hammond and Helenius 1994). Hence, we studied by confocal microscopy the intracellular distribution of AcGFP-tagged $\mathrm{A} 1 * 00101$ and $\mathrm{B} * 01202$ (Fig. 3) as well as $\mathrm{B}^{*} 06002$ and $\mathrm{I}^{*} 010201$ (not shown) by 


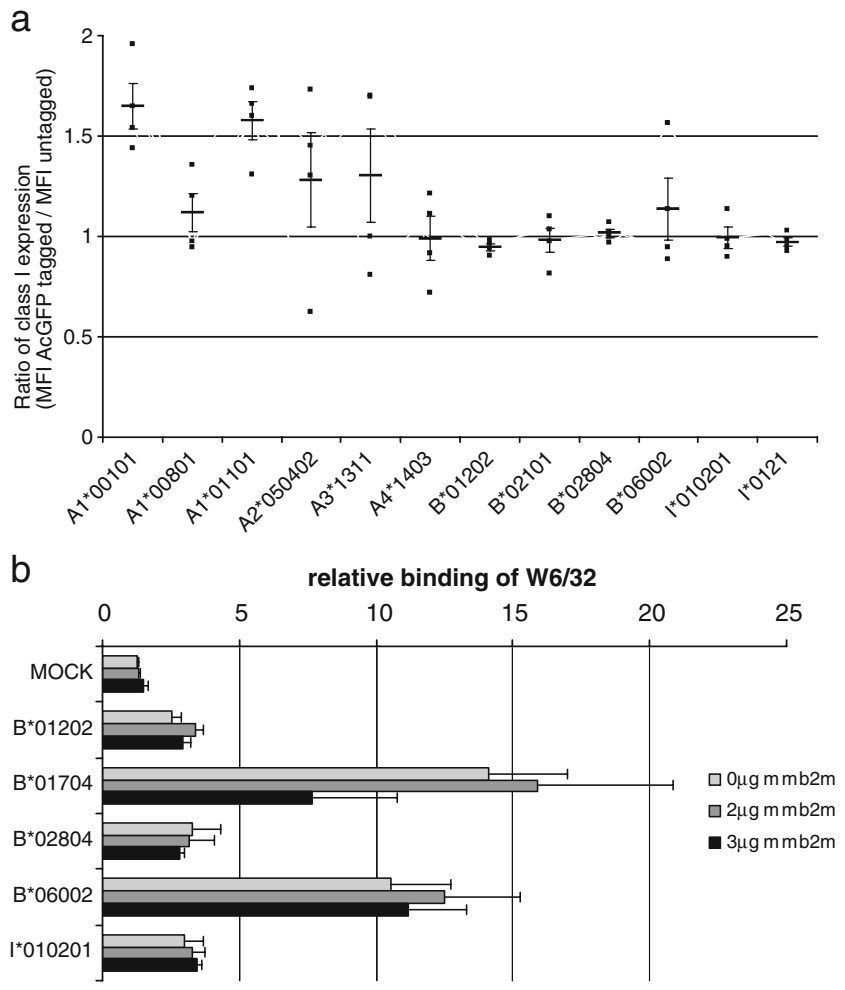

Fig. 2 AcGFP-tagging and presence of rhesus macaque $\beta 2$ microglobulin do not affect the surface expression of rhesus macaque MHC class I molecules. a Constructs coding for Mamu-A1*00101, $\mathrm{A} 1 * 00801, \mathrm{~A} 1 * 01101, \mathrm{~A} 2 * 050402, \mathrm{~A} 3 * 1311, \mathrm{~A} 4 * 1403, \mathrm{~B} * 01202$, $\mathrm{B}^{*} 02101, \mathrm{~B}^{*} 02804, \mathrm{~B}^{*} 06002, \mathrm{I}^{*} 010201$ and $\mathrm{I}^{*} 0121$ either with or without C-terminal AcGFP were transiently transfected in K562 cells. Surface expression was measured 2 days post-transfection by flow cytometry with W6/32 and polyclonal PeCy5-coupled goat anti-mouse antibody. The MFI ratios of AcGFP-tagged versus untagged MHC class I of four independent experiments are indicated. Horizontal bars indicate the average and vertical bars denote SEM. b K562 cells were transiently transfected with AcGFP-tagged Mamu-B constructs along with either none (control), $2 \mu \mathrm{g}$, or $3 \mu \mathrm{g}$ of rhesus macaque $\beta 2$ microglobulin expression construct $(\mathrm{mmB} 2 \mathrm{M})$. Expression of $\mathrm{mmB} 2 \mathrm{M}$ was controlled in parallel experiments with anti-V5 tagspecific antibody. The average of three independent experiments is shown and error bars denote SEM

co-staining transfected K562 cells with markers for intracellular compartments. For both Mamu-A1*00101 (Fig. 3) and Mamu-B*06002 (not shown) partial co-localisation with Golgi marker GM130 and with late endosomal/ lysosomal marker LAMP-1 can be seen, but is not visible with ER marker calnexin, indicating transport through Golgi apparatus, recycling of $\mathrm{A} 1 * 00101$ and $\mathrm{B} * 06002$ from cell surface to lysosomes, and fast egress from ER, respectively. Such intracellular staining pattern was expected for cell surface-expressed MHC class I molecules (Gromme et al. 1999). The intracellularly expressed MHC class I molecules B*01202 (Fig. 3) and Mamu-I*010201 (data not shown) show the opposite pattern: co-localisation with calnexin, but not with GM130 or LAMP-1. We conclude from these findings that the intracellularly expressed Mamu-B and Mamu-I molecules are retained within the ER.

Differential expression patterns can be assigned to the $\alpha 1$ and $\alpha 2$ domains

Intracellular localisation of MHC class I molecules was described for HLA-C and is due to inefficient assembly,
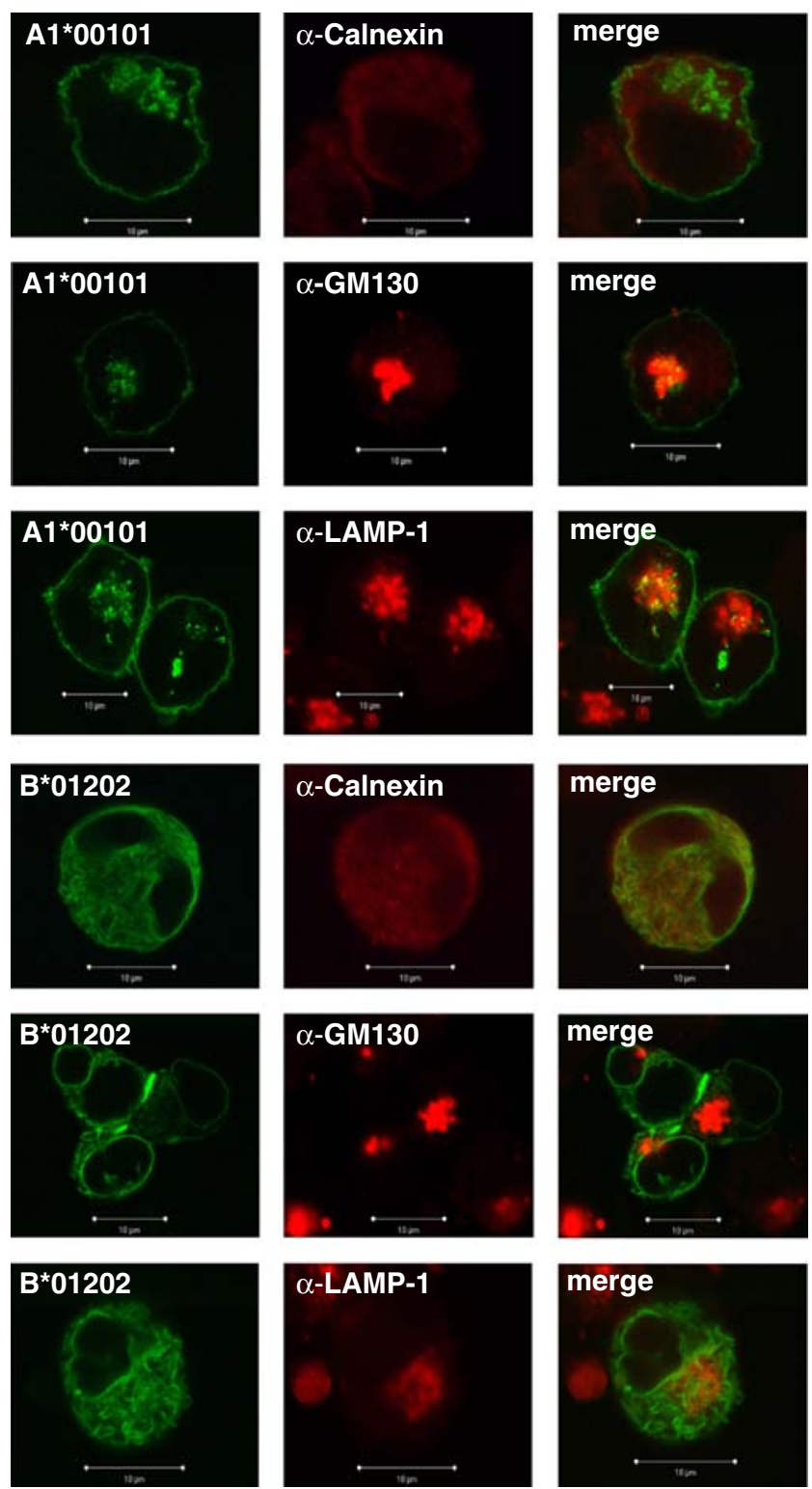

Fig. 3 Co-localisation of AcGFP-tagged Mamu-A1*00101 and Mamu-B*01202 molecules with organelle-specific markers by confocal microscopy ( $\times 63$ magnification). Left panels show AcGFP, middle panels show markers for ER (calnexin), Golgi apparatus (GM130), late endosomal/lysosomal compartment (LAMP-1) and right panels show merge. Co-localisation of class $I$ and the marker protein is shown in yellow. Note that co-localisation of $\mathrm{B}^{*} 01202$ and calnexin is evident, but the yellow colour is pale 
Fig. 4 Subcellular localization of Mamu-B molecules can be assigned to the $\alpha 1$ and $\alpha 2$ domains. a Scheme of chimeric constructs. The chimeric constructs of Mamu-I*010201 and Mamu-B*06002 are named according to the exchanged domains $(\alpha 1, \alpha 2$ or $\alpha 1$ and $\alpha 2$ domains together). $S P$ signal peptide, $T M / C T$ transmembrane region and cytoplasmic tail, AcGFP Aequorea coerulescens green fluorescent protein. b K562 cells were transiently transfected and analysed with W6/32 monoclonal antibody and secondary goat anti-mouse Pe-Cy5-coupled antibody 2 days post-transfection by flow cytometry. Lower panel shows confocal laser microscopy $(\times 63$ magnification) merged images of AcGFP-tagged Mamu class I molecules (green) and W6/32 (red). Numbering allows for rapid identification of the respective constructs shown in (a). All experiments were performed at least three times with similar results and a representative experiment is shown a
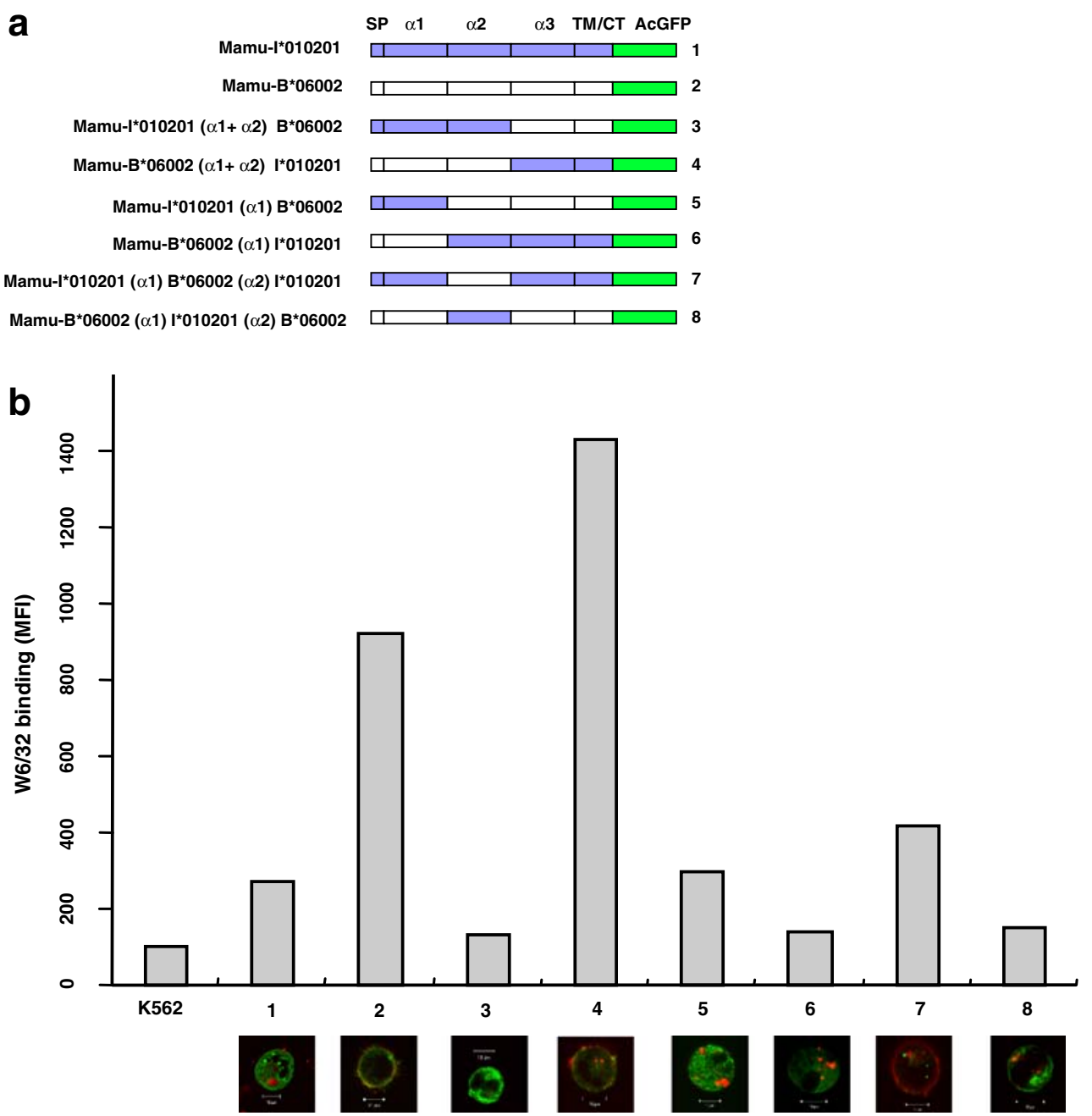

slower intracellular transport and stronger association with the TAP complex compared to HLA-A and HLA-B (Neisig et al. 1998; Sibilio et al. 2008). Mutation of single amino acid residues revealed that cysteine residues in the cytoplasmic tail of certain HLA-B and HLA-C alleles are crucial for cell surface expression (Gruda et al. 2007). However, such amino acid residues or corresponding differences in cytoplasmic tails of 'high' or 'low' cell surface expressors are not found in Mamu-B molecules (not shown), suggesting that the extracellular part, in particular the $\alpha 1$ and $\alpha 2$ domains, might be responsible for differential expression patterns of Mamu-B molecules. We tested this hypothesis by using chimeric expression constructs (Fig. 4a) where we exchanged exons 2 and 3 and chose Mamu-B*06002 and Mamu-I*010201 as representatives of high cell surface and high intracellular expression, respectively. The chimeric molecule carrying the $\alpha 1$ and $\alpha 2$ domains of $\mathrm{B}^{*} 06002$ and the $\mathrm{C}$-terminal part of $\mathrm{I}^{*} 010201$ was well expressed on the cell surface like the parental $\mathrm{B}^{*} 06002$ (Fig. 4b). Thus, the expression pattern is determined by the $\alpha 1$ and $\alpha 2$ domains and not by the $\alpha 3$ domain or transmembrane and cytoplasmic regions. Similar results were obtained for the chimaera with the $\alpha 1$ and the $\alpha 2$ domains derived from $I^{*} 010201$ and the C-terminus from $\mathrm{B}^{*} 06002$, showing the same expression pattern as the parental I*010201 (Fig. 4b). The observed expression patterns can neither be assigned to the $\alpha 1$ nor the $\alpha 2$ domain alone. Exchange of either domain alone resulted in a substantial reduction of MHC class I cell surface expression in almost all cases, which might be due to lower stability of chimeric molecules. An exception is the $\alpha 2$ domain of $\mathrm{B}^{*} 06002$ that conferred a slightly enhanced cell surface expression in the context of an I*010201 framework, although still not achieving parental B*06002 MFI values (Fig. 4b). We conclude from these observations that the peptide-binding groove is responsible for the observed differences in subcellular localization and hypothesise that Mamu-A and Mamu-B molecules with low or nearly absent cell surface expression are not efficiently loaded with peptides and therefore, are retained 
in the ER. These Mamu-B molecules might preferentially bind rare peptides that are available only in certain physiological or pathological situations, finally resulting in ER release and cell surface expression, analogous to what was previously demonstrated for mouse non-classical H2-M3 (Chiu et al. 1999). In accordance with this hypothesis, the intracellularly expressed Mamu-B*012 is known for its rather uncommon peptide-binding anchor residue at position P2 (Hickman-Miller et al. 2005). However, even very small amounts of cell-surface MHC class I can be sufficient for CTL recognition as demonstrated in a recent paper by Dressel and colleagues (2009), showing highly efficient CTL-mediated and peptidedependent killing of multipotent adult germ-line stem cells that appeared MHC class I negative in flow cytometry analysis. Hence, also rhesus macaque MHC class I molecules with limited amounts on the cell surface might contribute to antigen presentation function. Indeed, the weakly expressed Mamu-B*012 was previously shown to present a SHIV-derived peptide to rhesus macaque CTLs (Voss and Letvin 1996). The obvious functional significance of lowly expressed MHC class I molecules raises an important immunological question: how can rhesus macaques back up a sufficiently broad $\mathrm{T}$ cell receptor repertoire during thymic selection despite facing a plethora of different MHC class I proteins? The key to that enigma may lie in macaques' strategy of MHC diversity: low allelic polymorphism and high haplotypic diversity in terms of copy number variations. This strategy fits well with the recent finding of inherited control of thymic selection by germ-line-encoded amino acids of the $\mathrm{T}$ cell receptor (Scott-Browne et al. 2009). A further explanation for occurrence of intracellular class I molecules of rhesus macaques might be adaptation to certain viral infections and viral subversions of the class I antigen presentation machinery by 'spamming' the ER with different types of MHC class I molecules, thereby reducing the probability of viral immune evasins to down-regulate classical rhesus macaque MHC class I proteins. Nevertheless, our data clearly demonstrate that many of the rhesus macaque class I molecules show an unexpected subcellular localization and this indicates functional diversification of the duplicated genes. Future functional studies have to clarify the role of the intracellularly expressed MHC class I proteins and should help to explain disease associations of certain rhesus macaque $M H C$ class I haplotypes.

Acknowledgements The authors are grateful to Nicole Otto for the expert technical assistance and the veterinary staff for drawing blood samples. This study was supported by the German Primate Centre. P. H.K. is a student of the Göttingen Graduate School of Neuroscience and Molecular Biology (GGNB). This work was in part supported by European Union grant 'EUPRIM-Net' (FP6 026155-I3) to L.W.
Open Access This article is distributed under the terms of the Creative Commons Attribution Noncommercial License which permits any noncommercial use, distribution, and reproduction in any medium, provided the original author(s) and source are credited.

\section{References}

Bijlmakers MJ, Ploegh HL (1993) Putting together an MHC class I molecule. Curr Opin Immunol 5:21-26

Blasky AJ, Karl JA, Wiseman RW, Read DS, O'Connor DH (2008) Rapid high-resolution MHC class I genotyping of Chinese rhesus macaques by capillary reference strand-mediated conformational analysis. Immunogenetics 60:575-584

Bonhomme M, Blancher A, Jalil MF, Crouau-Roy B (2007) Factors shaping genetic variation in the MHC of natural non-human primate populations. Tissue Antigens 70:398-411

Bonhomme M, Doxiadis GG, Heijmans CM, Vervoort V, Otting N, Bontrop RE, Crouau-Roy B (2008) Genomic plasticity of the immune-related Mhc class I B region in macaque species. BMC Genomics 9:514

Boyson JE, Iwanaga KK, Golos TG, Watkins DI (1997) Identification of a novel MHC class I gene, Mamu-AG, expressed in the placenta of a primate with an inactivated $G$ locus. J Immunol 159:3311-3321

Chiu NM, Chun T, Fay M, Mandal M, Wang CR (1999) The majority of H2-M3 is retained intracellularly in a peptide-receptive state and traffics to the cell surface in the presence of $\mathrm{N}$-formylated peptides. J Exp Med 190:423-434

Daza-Vamenta R, Glusman G, Rowen L, Guthrie B, Geraghty D (2004) Genetic divergence of the rhesus macaque major histocompatibility complex. Genome Res 14:1501-1515

Dressel R, Guan K, Nolte J, Elsner L, Monecke S, Nayernia K, Hasenfuss G, Engel W (2009) Multipotent adult germ-line stem cells, like other pluripotent stem cells, can be killed by cytotoxic $\mathrm{T}$ lymphocytes despite low expression of major histocompatibility complex class I molecules. Biol Direct 4:31

Garstka M, Borchert B, Al-Balushi M, Praveen PV, Kuhl N, Majoul I, Duden R, Springer S (2007) Peptide-receptive major histocompatibility complex class I molecules cycle between endoplasmic reticulum and cis-Golgi in wild-type lymphocytes. J Biol Chem 282:30680-30690

Goulder PJ, Watkins DI (2008) Impact of MHC class I diversity on immune control of immunodeficiency virus replication. Nat Rev Immunol 8:619-630

Gromme M, Uytdehaag FG, Janssen H, Calafat J, van Binnendijk RS, Kenter MJ, Tulp A, Verwoerd D, Neefjes J (1999) Recycling MHC class I molecules and endosomal peptide loading. Proc Natl Acad Sci U S A 96:10326-10331

Gruda R, Achdout H, Stern-Ginossar N, Gazit R, Betser-Cohen G, Manaster I, Katz G, Gonen-Gross T, Tirosh B, Mandelboim O (2007) Intracellular cysteine residues in the tail of MHC class I proteins are crucial for extracellular recognition by leukocyte Iglike receptor 1. J Immunol 179:3655-3661

Hammond C, Helenius A (1994) Quality control in the secretory pathway: retention of a misfolded viral membrane glycoprotein involves cycling between the ER, intermediate compartment, and Golgi apparatus. J Cell Biol 126:41-52

Hickman-Miller HD, Bardet W, Gilb A, Luis AD, Jackson KW, Watkins DI, Hildebrand WH (2005) Rhesus macaque MHC class I molecules present HLA-B-like peptides. J Immunol 175:367375

Karl JA, Wiseman RW, Campbell KJ, Blasky AJ, Hughes AL, Ferguson B, Read DS, O'Connor DH (2008) Identification of 
MHC class I sequences in Chinese-origin rhesus macaques. Immunogenetics 60:37-46

Kulski JK, Anzai T, Shiina T, Inoko H (2004) Rhesus macaque class I duplicon structures, organization, and evolution within the alpha block of the major histocompatibility complex. Mol Biol Evol 21:2079-2091

Loffredo JT, Friedrich TC, Leon EJ, Stephany JJ, Rodrigues DS, Spencer SP, Bean AT, Beal DR, Burwitz BJ, Rudersdorf RA, Wallace LT, Piaskowski SM, May GE, Sidney J, Gostick E, Wilson NA, Price DA, Kallas EG, Piontkivska H, Hughes AL, Sette A, Watkins DI (2007) CD8+ T cells from SIV elite controller macaques recognize Mamu-B*08-bound epitopes and select for widespread viral variation. PLoS ONE 2:e1152

Mothe BR, Sidney J, Dzuris JL, Liebl ME, Fuenger S, Watkins DI, Sette A (2002) Characterization of the peptide-binding specificity of Mamu-B*17 and identification of Mamu-B*17-restricted epitopes derived from simian immunodeficiency virus proteins. J Immunol 169:210-219

Myers NB, Wormstall E, Hansen TH (1996) Differences among various class I molecules in competition for beta $2 \mathrm{~m}$ in vivo. Immunogenetics 43:384-387

Nei M, Gu X, Sitnikova T (1997) Evolution by the birth-and-death process in multigene families of the vertebrate immune system. Proc Natl Acad Sci U S A 94:7799-7806

Neisig A, Melief CJ, Neefjes J (1998) Reduced cell surface expression of HLA-C molecules correlates with restricted peptide binding and stable TAP interaction. J Immunol 160:171179

Otting N, Heijmans CM, Noort RC, de Groot NG, Doxiadis GG, van Rood JJ, Watkins DI, Bontrop RE (2005) Unparalleled complexity of the MHC class I region in rhesus macaques. Proc Natl Acad Sci USA 102:1626-1631
Otting N, de Vos-Rouweler AJ, Heijmans CM, de Groot NG, Doxiadis GG, Bontrop RE (2007) MHC class I A region diversity and polymorphism in macaque species. Immunogenetics 59:367-375

Otting N, Heijmans CM, van der Wiel M, de Groot NG, Doxiadis GG, Bontrop RE (2008) A snapshot of the Mamu-B genes and their allelic repertoire in rhesus macaques of Chinese origin. Immunogenetics 60:507-514

Robinson J, Marsh SG (2007) IPD: the Immuno Polymorphism Database. Methods Mol Biol 409:61-74

Sauermann U, Siddiqui R, Suh YS, Platzer M, Leuchte N, Meyer H, Mätz-Rensing K, Stoiber H, Nürnberg P, Hunsmann G, StahlHennig C, Krawczak M (2008) Mhc class I haplotypes associated with survival time in simian immunodeficiency virus (SIV)infected rhesus macaques. Genes Immun 9:69-80

Scott-Browne JP, White J, Kappler JW, Gapin L, Marrack P (2009) Germline-encoded amino acids in the alphabeta T-cell receptor control thymic selection. Nature 458:1043-1046

Sibilio L, Martayan A, Setini A, Lo Monaco E, Tremante E, Butler RH, Giacomini P (2008) A single bottleneck in HLA-C assembly. J Biol Chem 283:1267-1274

Urvater JA, Otting N, Loehrke JH, Rudersdorf R, Slukvin II, Piekarczyk MS, Golos TG, Hughes AL, Bontrop RE, Watkins DI (2000) Mamu-I: a novel primate MHC class I B-related locus with unusually low variability. J Immunol 164:1386-1398

Vierboom MP, Jonker M, Bontrop RE, Hart B (2005) Modeling human arthritic diseases in nonhuman primates. Arthritis Res Ther 7:145-154

Voss G, Letvin NL (1996) Definition of human immunodeficiency virus type 1 gp120 and gp41 cytotoxic T-lymphocyte epitopes and their restricting major histocompatibility complex class I alleles in simian-human immunodeficiency virus-infected rhesus monkeys. J Virol 70:7335-7340 